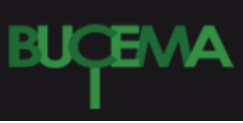

Bulletin du centre d'études médiévales d'Auxerre | BUCEMA

Hors-série $n^{\circ} 6 \mid 2013$

Autour du cloître : les chapelles Notre-Dame et les accès au chapitre

\title{
Payerne VD, Abbatiale. Kapitelsaal und Marienkapelle - Stand der Forschung
}

\section{Guido Faccani}

\section{(2) OpenEdition \\ Journals}

Édition électronique

URL : https://journals.openedition.org/cem/12907

DOI : $10.4000 /$ cem. 12907

ISSN : 1954-3093

Éditeur

Centre d'études médiévales Saint-Germain d'Auxerre

Référence électronique

Guido Faccani, « Payerne VD, Abbatiale. Kapitelsaal und Marienkapelle - Stand der Forschung », Bulletin du centre d'études médiévales d'Auxerre | BUCEMA [En ligne], Hors-série n 6 | 2013, mis en ligne le 25 avril 2013, consulté le 03 mars 2023. URL : http://journals.openedition.org/cem/12907 ; DOI : https://doi.org/10.4000/cem.12907

Ce document a été généré automatiquement le 3 mars 2023.

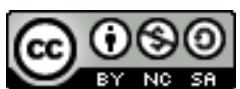

Creative Commons - Attribution - Pas d'Utilisation Commerciale - Partage dans les Mêmes Conditions 4.0 International - CC BY-NC-SA 4.0

https://creativecommons.org/licenses/by-nc-sa/4.0/ 


\title{
Payerne VD, Abbatiale. Kapitelsaal und Marienkapelle - Stand der Forschung
}

\author{
Guido Faccani
}

1 Die Forschung über die Abbatiale von Payerne (Abb.1) hat im vergangenen halben Jahrhundert zahlreiche neue und unerwartete Resultate erbracht. Die in der schriftlichen Überlieferung bestehenden grossen Lücken für die frühe Geschichte und u.a. die daraus folgenden Fragen der Bauchronologie vermochten Archäologie, Architektur- und Kunstgeschichte aber nicht zu klären.

2 Der Kapitelsaal und eine nach cluniazensischem Vorbild damit zusammenhängende Marienkapelle waren bislang noch nicht Gegenstand der Forschung. Die folgenden Ausführungen sind dementsprechend als erste Annäherung an die beiden Themenfelder zu verstehen.

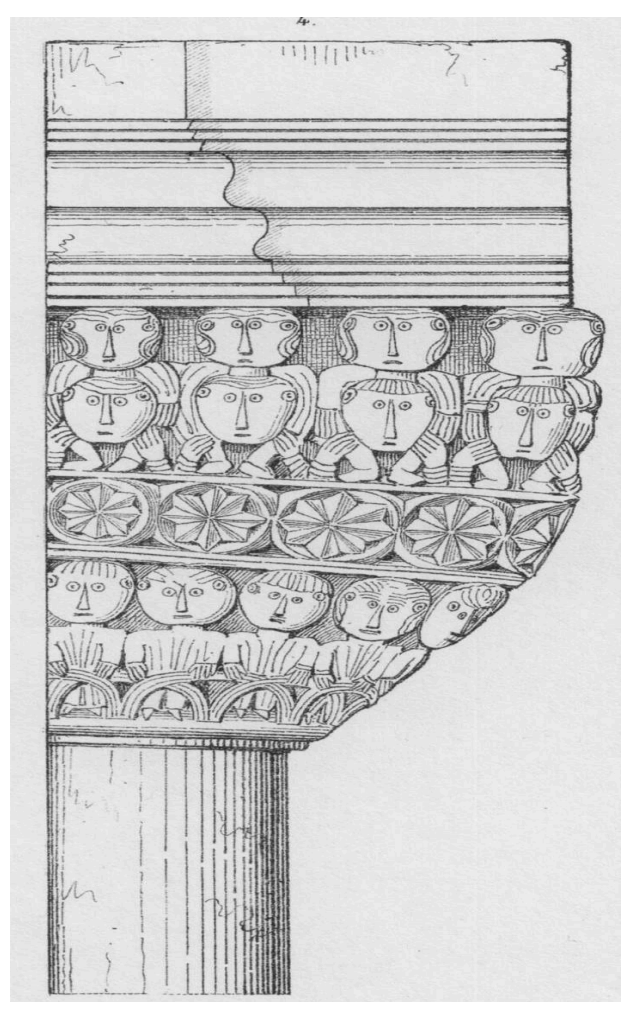


Fig. 1 - Payerne, Abbatiale. Zustand 1920. Gegen Nordwesten. Bild Louis Bosset.

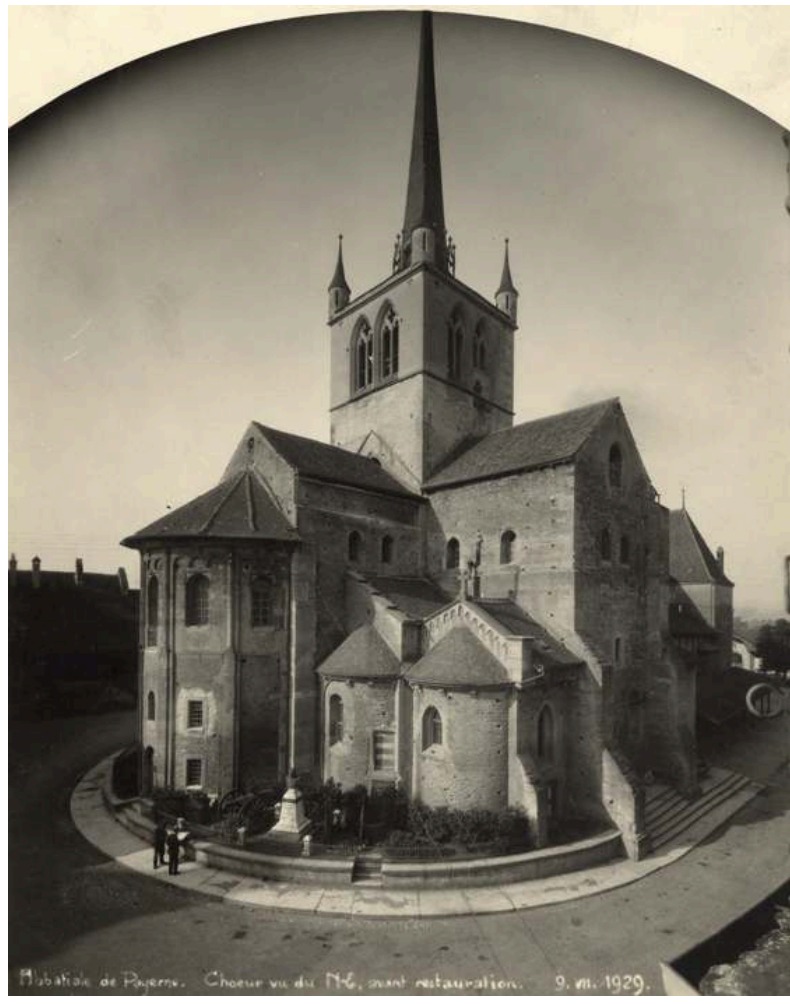

Einleitung - introduction 
Fig. 2 - Payerne. Blau: Broye. Hellgrün: mittelalterliche Stadtfläche. Dunkelgrün: Erhebung im Zentrum der Stadt. Rot: Kirchen - unten links Katholische Kirche, Mitte Abbatiale mit Kreuzgang, rechts reformierte Paroissiale. Norden oben. Bild: GIS-VD und Autor.

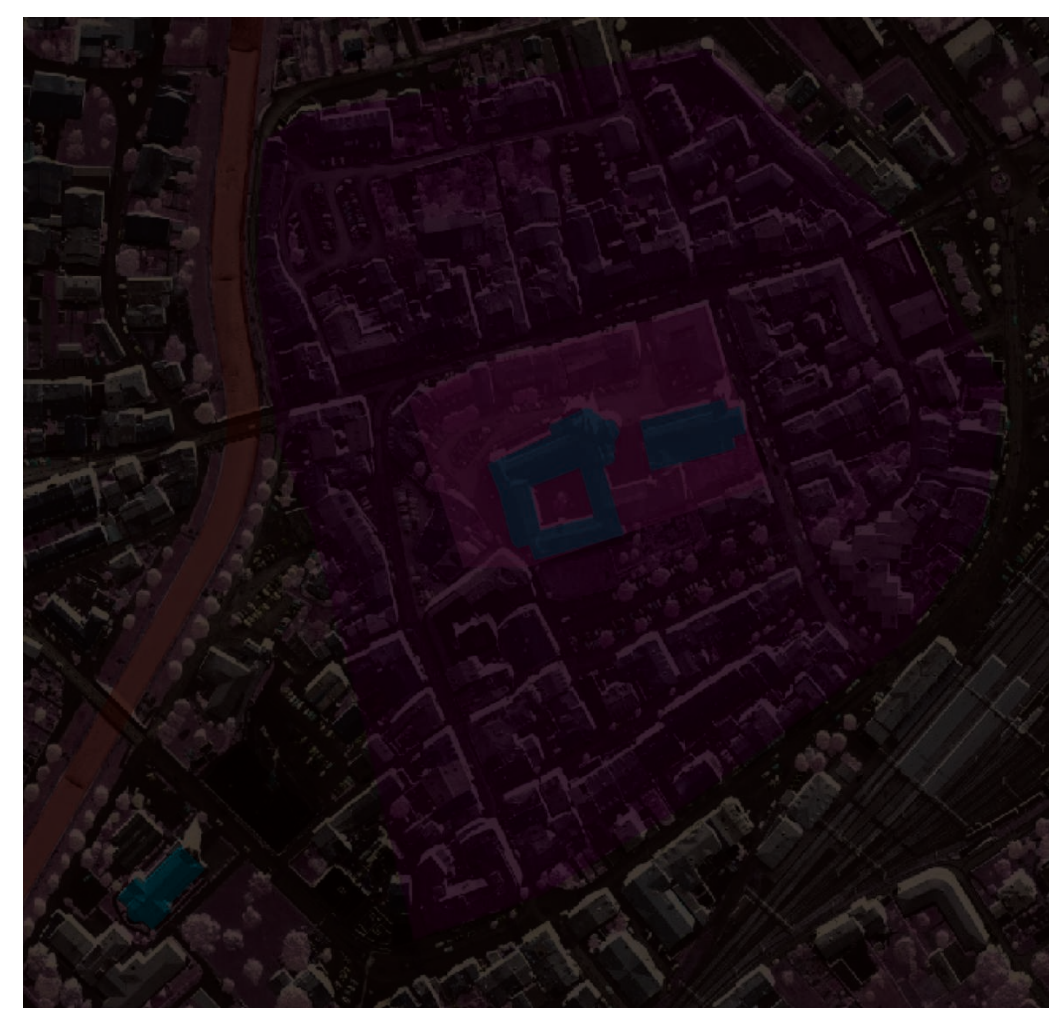

3 In Payerne dienen heute zwei Kirchen den beiden grossen Konfessionen zum regelmässigen Gottesdienst (Abb. 2). ${ }^{1}$ Das katholische Gotteshaus Ste-MarieImmaculée, 1928/29 erbaut im neuromanischen Stil, liegt an der südwestlichen Grenze der mittelalterlichen Stadt. Die reformierte Paroissiale (Pfarrkirche), im Mittelalter Maria geweiht, steht am östlichen Rand der Erhebung im Zentrum der Altstadtfläche. ${ }^{2}$ Sie erscheint 1183 erstmals in den Quellen, ${ }^{3}$ ihre sichtbare Architektur ist aber rund 100 Jahre jünger (Chor um 1300, Schiff um 1335). Unmittelbar westlich der Paroissiale steht das bedeutendste Baudenkmal von Payerne, die Maria dedizierte Abbatiale (Abteikirche, Abb.1, 3) ${ }^{4}$. Die ehemalige cluniazensische Klosterkirche ist im Wesentlichen ein Bau des 11. bzw. frühen 12. Jh. Nachdem sie bei der Reformation im Jahr 1536 profaniert und für verschiedenste Zwecke umgenutzt worden ist - vom Speicher über die Kaserne bis zum Gefängnis -, wird sie seit den 1960er Jahren hauptsächlich als Museum und Konzertsaal, gelegentlich aber auch für spezielle Gottesdienste genutzt. Von den Konventbauten ist der Ostflügel in seiner romanischen Substanz in wichtigen Teilen noch erhalten, während der Süd- und der Westflügel im 17. bzw. 19. Jh. Neubauten weichen mussten. ${ }^{5}$

\section{Zur Forschungsgeschichte}

\section{Archäologie und Bauuntersuchungen in Abbatiale und Paroissiale}

Die archäologische Erforschung der Abbatiale begann vor knapp 200 Jahren. Erste Ausgrabungen führte der Offizier François-Rodolphe de Dompierre (1775-1844) zwischen 1817 und 1818 in Vorhalle, Chor und Seitenkapellen durch. ${ }^{6}$ Ein Jahrhundert 
später begannen unter Architekt Louis Bosset ${ }^{7}$ (1880-1950) im Jahr 1920 tiefgreifende Restaurierungskampagnen. Sie prägen die Klosterkirche und die ehemaligen Konventbauten bis heute. Auf Bosset folgte ab 1951 bis in die 1980er Jahre Pierre Margot (1922-2011) als leitender Architekt. ${ }^{8}$ Unter Bosset und Margot wurde der gesamte Innenraum der Abbatiale und grosse angrenzende Flächen archäologisch untersucht.

Fig. 3 - Payerne, schematische Darstellung der Grabungsflächen im Gebiet von Abbatiale und Paroissiale.1: Abbatiale. 2: Osttrakt der Konventgebäude. 3 Cour du Château. 4: Neubau des mittleren 17. Jh. anstelle des Südtraktes. 5: Neubau des 19. Jh. anstelle des Westtraktes. 6: Place du Tribunal. 7: Paroissiale. Bild Archéotech und Autor.

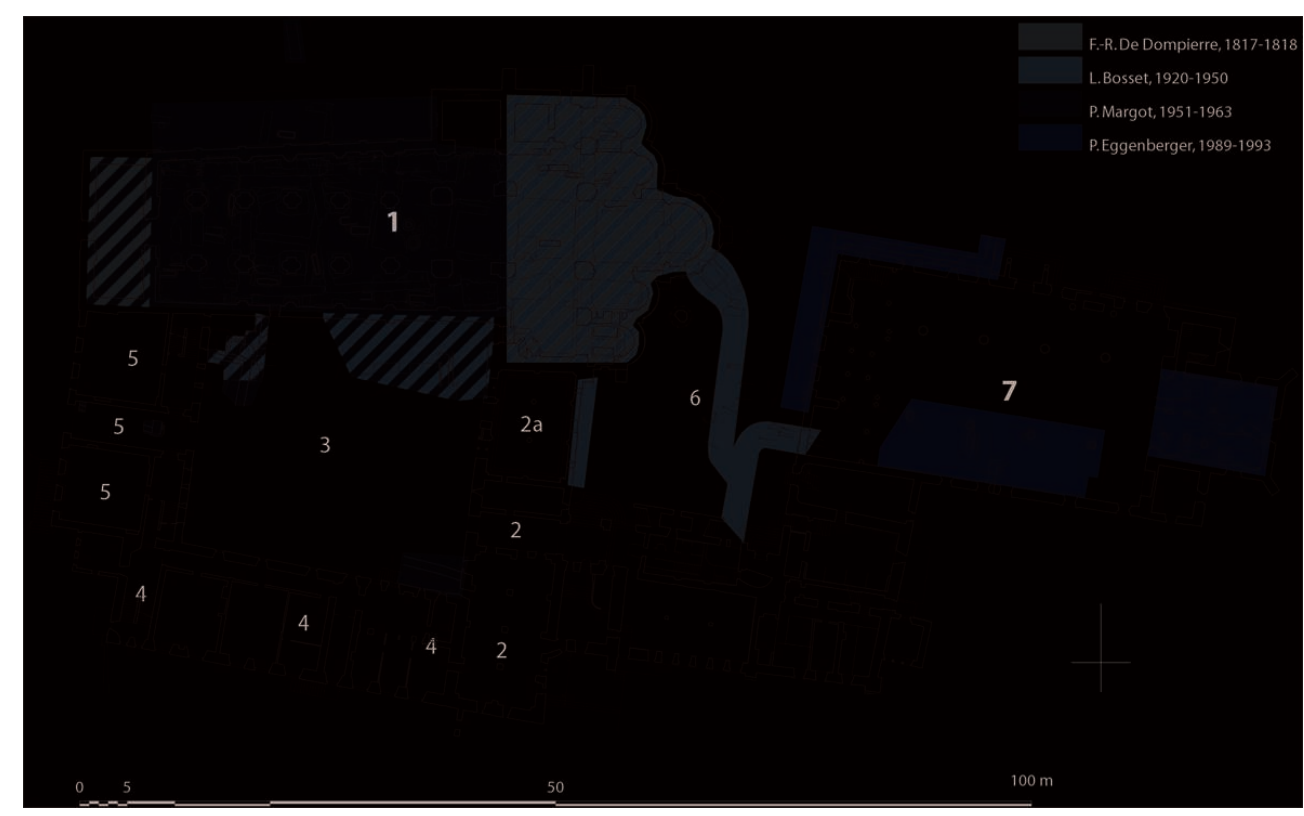

5 Ein Hauptergebnis von Bosset war die Freilegung der Kreuzgangreste in der Cour du Château sowie die Entdeckung eines Friedhofes auf der Place du Tribunal, d.h. östlich der Abbatiale. Der Friedhof zog unter der Paroissiale weiter nach Osten. In der Abbatiale deckte Bosset in den äusseren Chorkapellen noch ungestörte Bestattungen und Sarkophage auf, Chorquadrat und Querhaus dagegen waren von de Dompierre fast vollständig leergeräumt. Margot dehnte die Ausgrabungen auf Vorhalle und Schiffe aus, legte den Kreuzgang ein zweites Mal partiell frei und untersuchte einen ca. vier Meter breiten Streifen aussen entlang der Nordschiffmauer. Margots Grabungsresultate belegen eine römische, kaiserzeitliche Architektur unbekannter Ausdehnung und ein darüber errichtetes, anders orientiertes Gebäude, das zweimal nach Westen erweitert worden war. Ebenfalls legte Margot mehr als 160 Bestattungen frei, vom Erdgrab bis zum Sarkophag. Eine detaillierte Bearbeitung der umfangreichen Dokumentation steht noch aus.

6 Seither fanden verschiedene kleine Interventionen statt, z.B. die zeichnerischen Neudokumentation des über der römischen Architektur errichteten Gebäudes durch den Schreibenden. ${ }^{9}$ Für die mittlerweile durch statische Probleme gefährdete Abbatiale und für den gesamten ehemaligen Klosterkomplex besteht schliesslich seit 2008 ein Bauprogramm zur Restaurierung. Die Leitung der Untersuchungen sowohl im Boden als auch am Aufgehenden obliegen dem Schreibenden. 
7 Während die Abbatiale auf eine lange Grabungsgeschichte zurückblicken kann, fanden in der Paroissiale erst in den Jahren von 1989 bis 1993 die ersten archäologischen Untersuchungen statt. Peter Eggenberger führte im Chor eine Flächengrabung und im Schiff Sondierungen durch. ${ }^{10}$ Während im Schiff, wo nicht in die Tiefen gegraben wurde, Mauerzüge ans Tageslicht kamen, deren Datierung und Funktion noch offen ist, deckten die Untersuchungen im Chor einen älteren Friedhof, Pfostengruben und Mauerzüge auf. Die gotischen Schiffmauern überlagern im Westen und Süden zudem ältere Mauern.

\section{Architektur- und Kunstgeschichte}

8 Während die Architektur der Paroissiale bislang kaum Beachtung fand, stammt die erste kritisch-wissenschaftliche Veröffentlichung über die Abbatiale aus dem Jahr 1853, verfasst vom Architekten Jean Daniel de Blavignac (1817-1876). ${ }^{11} \mathrm{Er}$ gab seinem Text zahlreiche Illustrationen bei, darunter ein Grossteil der figürlichen Kapitelle und Konsolen.

Fig. 4 - Payerne, Abbatiale. Eckkapitell im Südquerhaus. Bild Blavignac 1853 (wie Anm. 11), pl. LVI. 4.

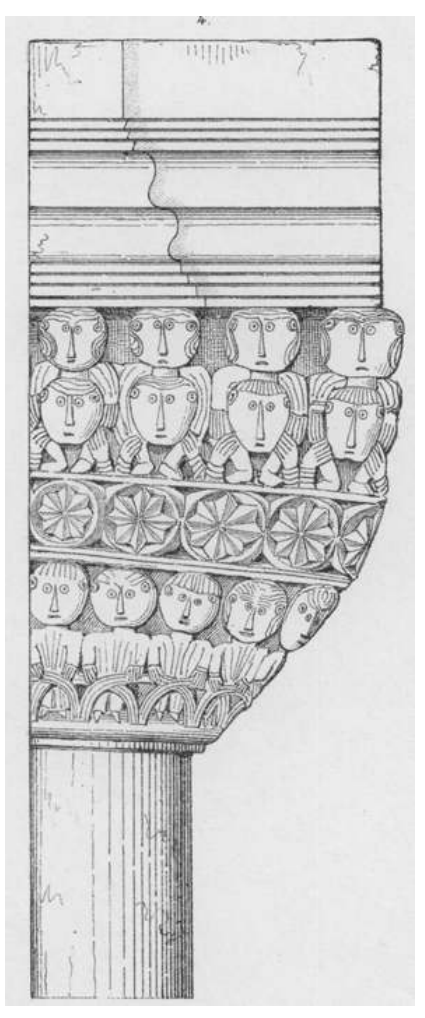

Für den weiteren baulichen Umgang mit der Abbatiale war aber die architekturhistorische Studie des Zürcher Kunstgeschichtsprofessors Johann Rudolf Rahn (1841-1912) entscheidend. ${ }^{12}$ Mit einem 1893 ins Französische übersetzten, reich illustrierten Text sensibilisierte Rahn die Fachwelt massgeblich für den romanischen Bau europäischer Bedeutung, was schliesslich 1899 zur Unterschutzstellung der Abbatiale führte. Louis Bosset publizierte 1917 im Rahmen einer knappen baugeschichtlichen Übersicht den ersten Bauphasenplan. ${ }^{13}$ 
Fig. 5 - Erster veröffentlichter Phasengrundriss der Abbatiale aus dem Jahr 1917 von Louis Bosset. Bild Bosset 1917 (wie Anm. 13).

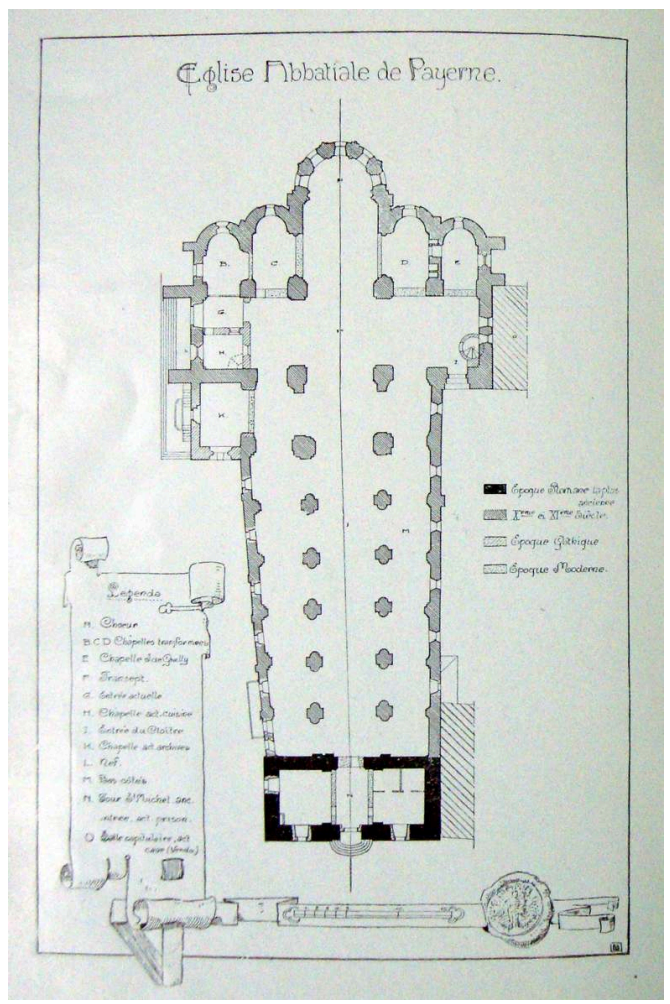

Die bislang einzige Gebäudemonographie erschien 1966, drei Jahre nach Abschluss der umfassenden Restaurierung. ${ }^{14}$ Darin sind zahlreiche Beiträge versammelt, archäologische, architekturhistorische und auch kunsthistorische, so die wegweisende Analyse der Kapitelle von Georges Zarnecki. ${ }^{15}$ Mit der 1970 veröffentlichten Habilitationsschrift Romainmôtier und Payerne von Hans Rudolf Sennhauser lag der Architektur-, aber auch der Bauforschung eine weitere detaillierte Studie über die Klosterkirche von Payerne vor. ${ }^{16}$ Die jüngste Ausgabe der populärwissenschaftlichen Kunstführer der Schweizerischen Gesellschaft für Kunstgeschichte aus dem Jahr 1991 verfasste Hans Rudolf Sennhauser. Der darin veröffentlichte farbige Bauphasenplan zeigt die Bauentwicklung der Kirche (Abb. 6). ${ }^{17}$ 
Fig. 6 - Payerne, Abbatiale. Bauphasenplan nach Sennhauser. Bild Sennhauser 1991 (wie Anm. 17), 6.

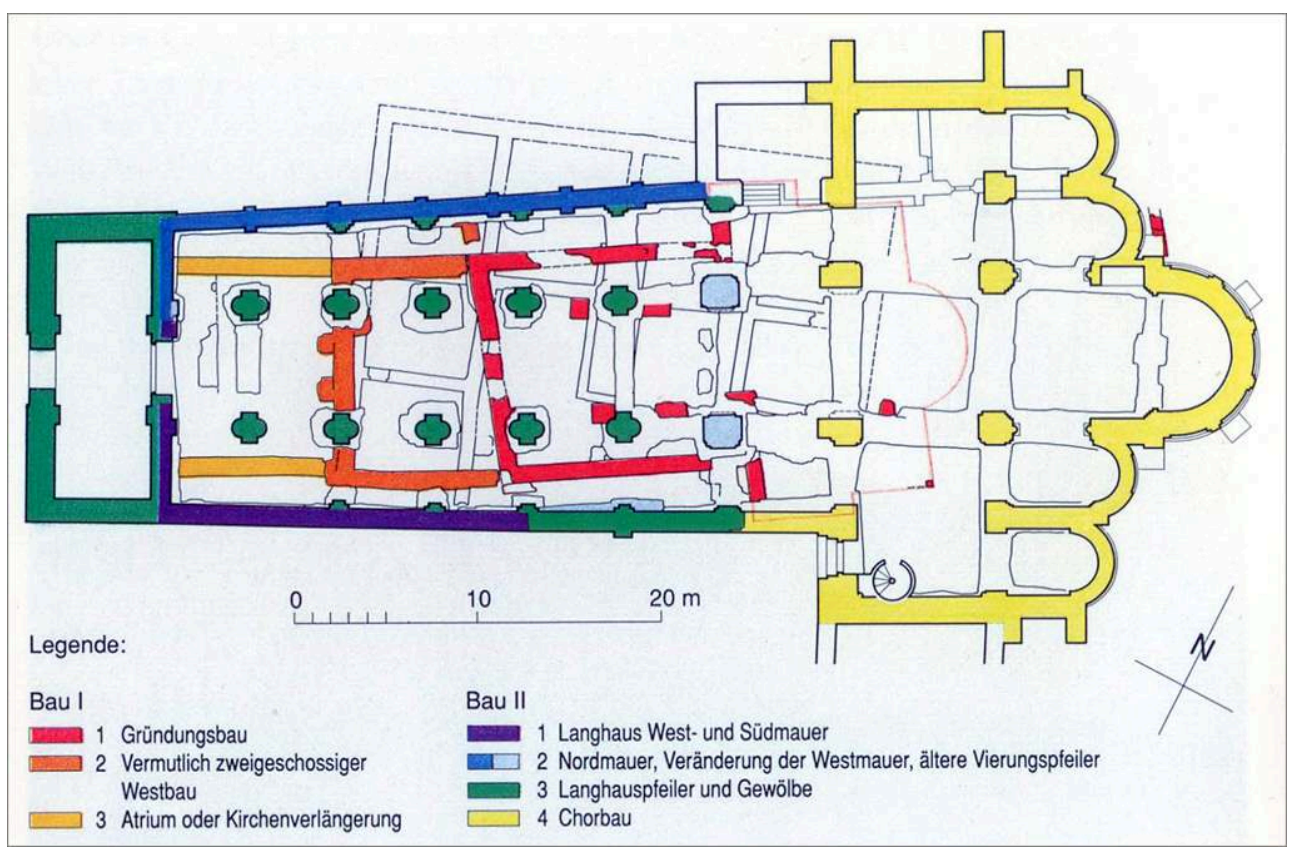

\section{Zu den Marienpatrozinien in Payerne}

Quellen zur Geschichte des Klosters von Payerne sind kaum vorhanden, insbesondere fehlen die Akten des ehemaligen Klosterarchives aus der Zeit zwischen dem 10. und mittleren 14. Jh. fast gänzlich. ${ }^{18}$ Ursache für die Lücken sind Brand und Plünderungen. ${ }^{19}$ Die dünne Quellenlage wird nicht verbessert durch den Umstand, dass etliche Ereignisse nur indirekt belegt sind, z.B. durch die vita des Abtes Odilo von Cluny oder ein Kartular des Lausanner Domkapitels. ${ }^{20}$ Zudem sind einige der frühen Schriftstücke Fälschungen des hohen und späten Mittelalters, so z.B. das in zwei Versionen erhaltene Testament der Königin Berta, das lange als Gründungsurkunde des Cluniazenserklosters galt. ${ }^{21}$

\section{Jahrhundert}

Die erste schriftliche Nachricht über ein Gotteshaus in Payerne entstammt einem Bericht über Bischof Marius (ca.530-593, im Amt spätestens seit 585) im hochmittelalterlichen Kartular des Domkapitels von Lausanne. Für den 24. Juni des Jahres 587 ist verzeichnet, ${ }^{22}$ dass Marius ein Marienoratorium geweiht habe, das er im gleichzeitig erbauten (wieder hergestellten?) Landgut in Payerne eingerichtet hatte: ,... Marius episcopus in honore sancte Marie genitricis Domini templum et villam Paterniacam in solo construxit proprio dedicavitque ..... ${ }^{23}$ Die Quelle gibt keine Auskunft über den genauen Standort des Oratoriums. Die Archäologie kann diese Unklarheit nicht beseitigen. U.a. ist die Ausdehnung des unter der Abbatiale nachgewiesenen römischen Anwesens (Abb. 7), das mit Marius' Landgut identisch sein könnte, unbekannt. 
Fig. 7 - Rekonstruktionsversuch des römischen Gebäudekomplexes. Dunkelbraun: erhalten. Hellbraun: rekonstruiert. Rote Zone: Ergrabene Mauern unbekannter Zeitstellung (Standort des Oratoriums von Marius?). Bild Archéotech und Autor.

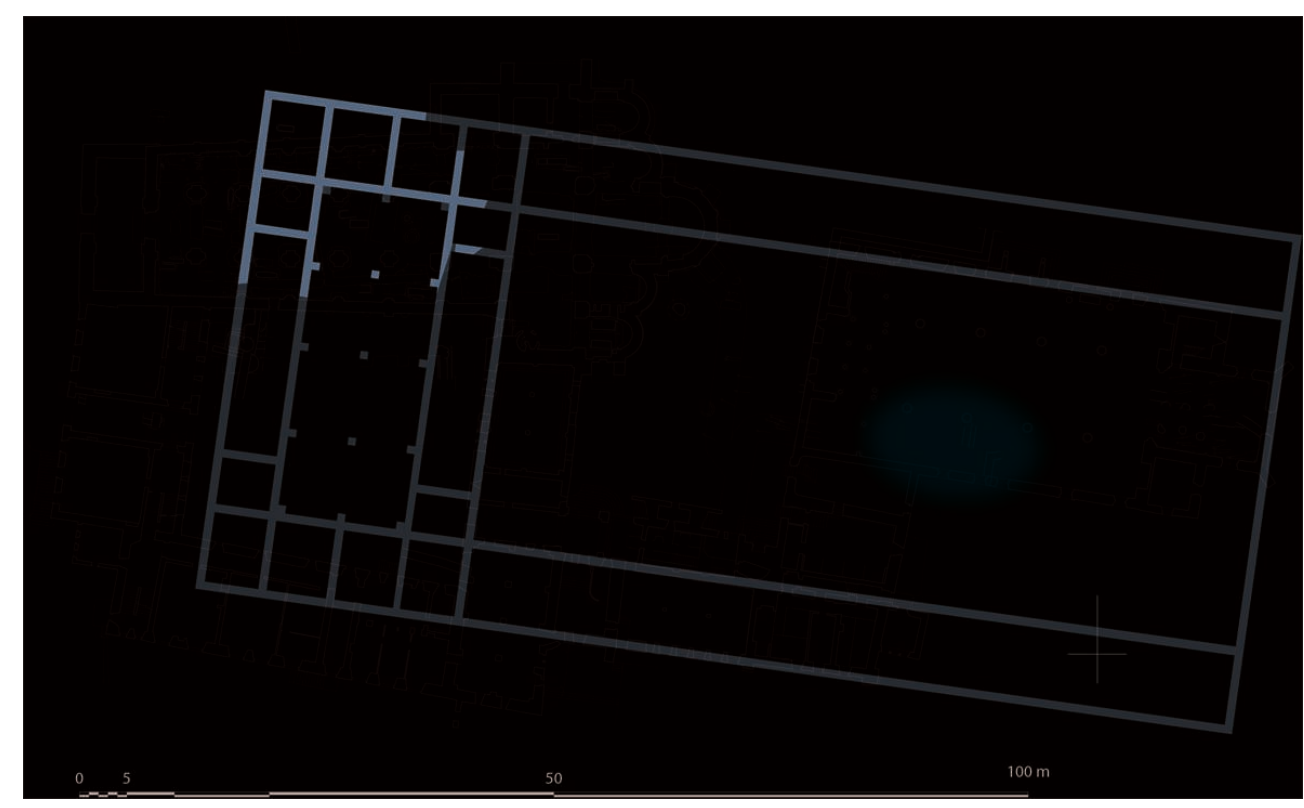

13 Aber auch das Marienpatrozinium kann nicht ohne weitere Informationen zur Klärung der Fragen herangezogen werden, da Abbatiale und Paroissiale gleichermassen Maria geweiht waren. Und keines der beiden Gotteshäuser greift z.B. im Rahmen eines Gründungsberichtes die Weihe Marius' auf, noch sind solche Berichte überhaupt bekannt. Möglicherweise lassen aber die Daten der Kirchweihfeste die Lokalisierung weiter einkreisen. Im 15. Jh. hatte man festgehalten, dass die Kirchweihe der Abbatiale am 17. April begangen wurde. Der Kirchweihe der Paroissiale gedachte man dagegen am 24. Juni, dem Tag, an dem auch Marius sein Oratorium im Jahr 587 weihte. ${ }^{24}$ Sofern diese spätmittelalterlichen Angaben tatsächlich im 6. Jh. wurzeln, wäre der Standort des Mariusoratoriums geklärt. Es läge unter der Pfarrkirche. Wenn man sich im Rahmen einer Arbeitshypothese noch einen Schritt weiter vorwagt, kann man auf die freilich undatierten Baureste hinweisen, welche Peter Eggenberger in im Südschiff der Paroissiale oberflächlich freilegte (Abb.7); sie liegen etwa auf der Mittelachse des rekonstruierten römischen Gebäudekomplexes. Künftige Ausgrabungen werden vielleicht einige Fragen klären.

\section{Jahrhundert}

14 Auf den Weihebericht von 587 folgt eine quellenlose Zeit von fast 400 Jahren. In der zweiten Hälfte des 10. Jh. setzt dann der Nachrichtenfluss unvermittelt wieder ein. Die Schriftquellen zeigen auf, dass nun das burgundische Königshaus Eigentümerin der vormals bischöflichen Güter von Payerne war.

15 Wichtig für das Verständnis der Anfänge des Klosters ist eine Urkunde, mit der König Konrad am 8. April 961 (in Lausanne) frühere Schenkungen an die Kirche von Payerne bestätigte. ${ }^{25}$ Daraus geht u.a. hervor, dass in Payerne ein Kanonikerstift bestand. ${ }^{26}$ Wann und von wem es gegründet wurde ist unbekannt. Dass das Stift aus einer älteren Gemeinschaft hervorging, welche das von Marius geweihte Marienoratorium betreut hatte, ist ebenso denkbar wie die Vermutung, die Gründung des Stiftes gehe auf die 
Burgunderkönigin Berta zurück. ${ }^{27}$ Berta wurde kurz vor dem 8. April 961 in der Stiftskirche begraben. ${ }^{28}$ Da keine Berichte über eine Translation der Gebeine von Berta bekannt sind, dürfte sie am Ort der nachmaligen Abbatiale bestattet worden sein und damit der Standort von Abbatiale und ehemaliger Stiftskirche gleichzusetzen sein. ${ }^{29}$ Doch angesichts der dürftigen Quellenlage ist dies nicht zu belegen. Immerhin geht aus der Urkunde König Konrads hervor, dass die Stiftskirche Maria geweiht war: „,.. aecclesiae sanctae Mariae Paterniensis..." ${ }^{30}$.

Erst nach Bertas Tod wurde das Stift von Payerne auf Initiative von Kaiserin Adelheid durch den Burgundischen König, ihren Bruder, an die Klostergemeinschaft und dessen damaligen Abt Majolus (im Amt 948/63-994) von Cluny übertragen - ein Akt, der nur indirekt im von Abt Odilo nach 999 verfassten Epitaph der Kaiserin überliefert ist. ${ }^{31}$ In einem Diplom Kaiser Ottos II. aus dem Jahr 973 ist festgehalten, dass die Majolus übereignete (Kloster-)Kirche von Payerne Maria geweiht war: „...sanctae aecclesiae semper virginis Mariae quae sita est Paterniaco...“. ${ }^{32}$ Dies ist der erste eindeutige Nachweis des Marienpatroziniums der Abbatiale.

\section{Jahrhundert}

Im ausgehenden 13. Jh. schliesslich wird das Marienpatrozinium der Paroissiale, die bereits 1183 in den Quellen fassbar ist, ein erstes Mal genannt. ${ }^{33}$ Die vom Kloster unabhängige Stadt formierte sich gerade in dieser Zeit. ${ }^{34}$ Die Paroissiale seht am Ort, wo bei anderen Cluniazenser-Konventen funeral konnotierte Marienkapellen stehen. Es bleibt zu prüfen, ob die heutige Paroissiale auf eine solche frühe romanische - oder noch ältere - Kapelle zurückgeht, die zuerst zum Kloster gehörte und erst mit der Trennung der Gewalten an die Stadt gelangte.

\section{Zu Entstehung und Aussehen des Kapitelsaals}

Vendo - so nannte man in Payerne zuletzt einen Raum, der südlich an das Querschiff anschloss und wo bis 1920 der Gemeindewein gelagert bzw. verkauft wurde(Abb. 8-10). Es handelt sich um den Kapitelsaal des ehemaligen Klosters. Heute ist der Raum Teil des Museums.

Fig. 8 - Payerne, Abbatiale. Ostflügel der Konventbauten im Jahr 1920. Gegen Osten. Links: Nach dem Öffnen des Rundbogenportals (provisorischer Bretterverschlag links). Rechts: Nach dem Abschlagen des Putzes. Bilder Louis Bosset.
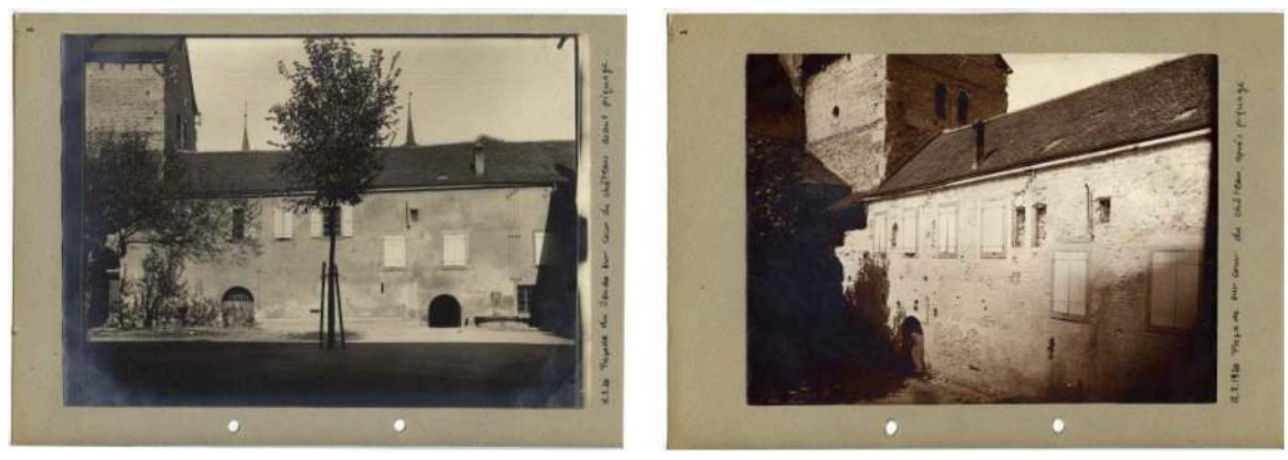
Fig. 9 - Payerne, Abbatiale. Ostflügel der Konventbauten im Jahr 1920. Links: Übersicht nach dem Abschlagen des Putzes, gegen Westen. Rechts: Drainage, in der Mitte der gegen den Kapitelsaal lehnende Pfeiler der Chapelle de Grailly, gegen Norden. Bilder Louis Bosset.
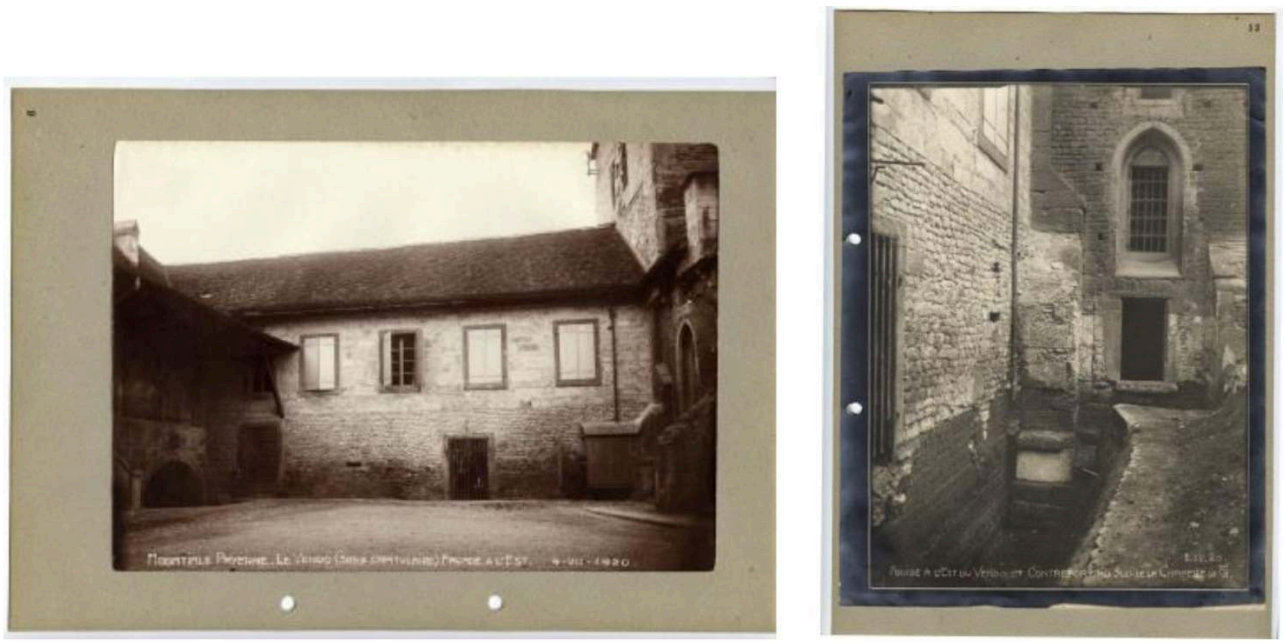

Fig. 10 - Payerne, Abbatiale. Kapitelsaal im Jahr 1920. Das Gewölbe stammt aus dem 14. Jh, die Wandbänke sind ev. älter. Links das wiedergeöffnete Rundbogenportal, rechts unten das Geländer der Treppe zum Osteingang. Gegen Nordwesten. Bild Louis Bosset.

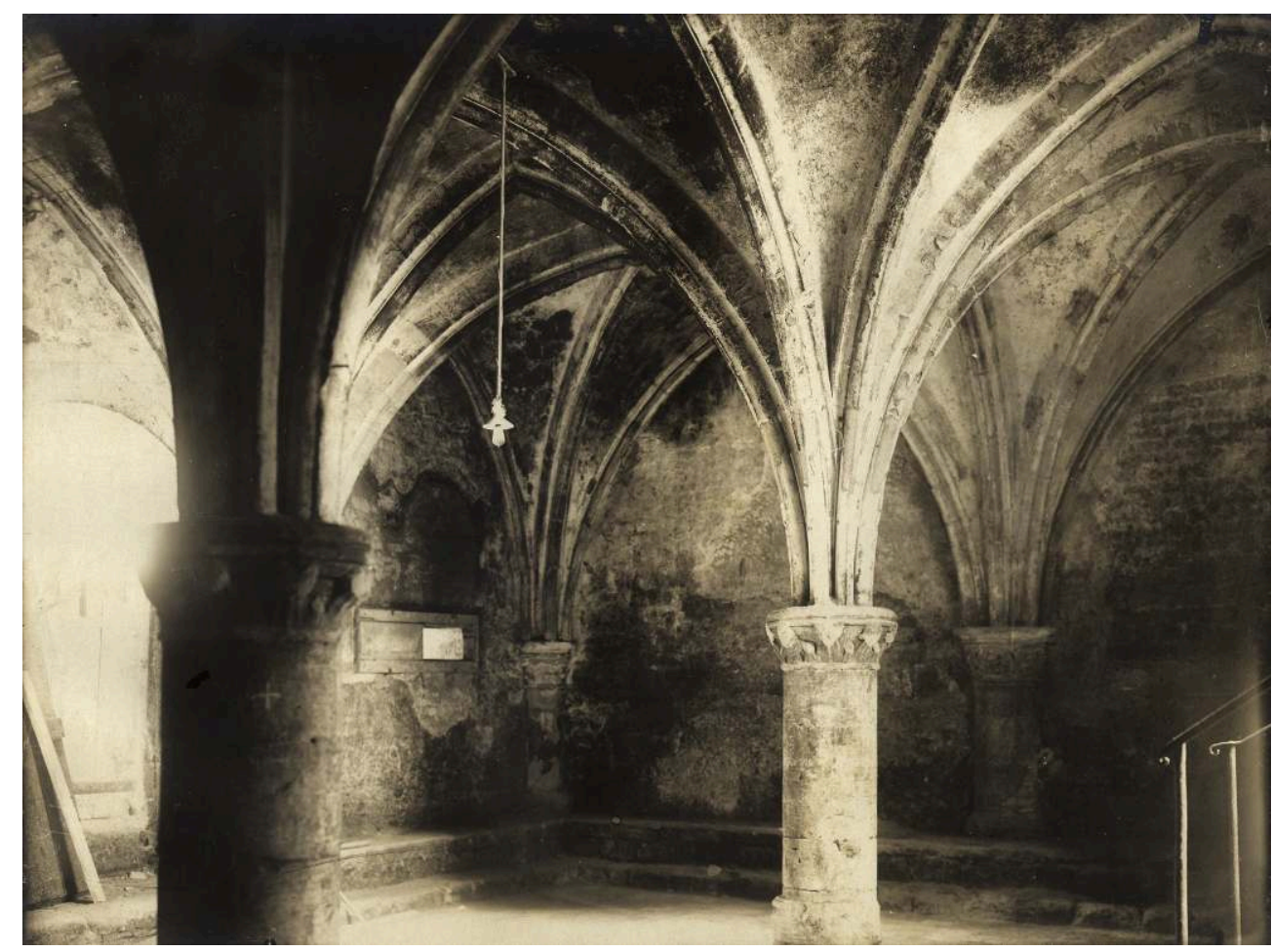

Als Louis Bosset im Frühjahr 1920 mit den Restaurierungsarbeiten an der Abbatiale begann, widmete er sich zuallererst dem Vendo. Er war bis 1920 von Osten über eine rechteckige Tür erschlossen, durch die man acht Stufen hinab in den Raum gelangte. Bosset liess zuerst die bis dahin verputzten Mauern steinsichtig freilegen. Da der Vendo im Innern sehr feucht war, erfolgte auf der Ostseite das Ausheben einer Drainage (Abb. 9). Auf der Westseite und im Innern initiierte Bosset kleinflächige Sondierungen. Seine Arbeiten hielt er schriftlich, zeichnerisch und fotografisch fest. ${ }^{35}$ Die Zeichnungen umfassen unmassstäbliche Skizzen (Abb. 11) sowie Pläne im Massstab 1:20 
(Abb.12). Die durchgeführten Untersuchungen verliefen äusserst erfolgreich, drei Hauptergebnisse sind zu nennen.

Fig. 11 - Payerne, Abbtiale. Sondierung von 1920 an der Grenze zwischen S-Arm Querschiff und Kapitelsaal. Links: nicht massstäbliche Skizze. Rechts Foto der skizzierten Sondierung. Gegen Osten. Bild links: Bosset 1920 (wie Anm. 35), 24. Bild rechts: Bosset 1920 (wie Anm. 35), 30.
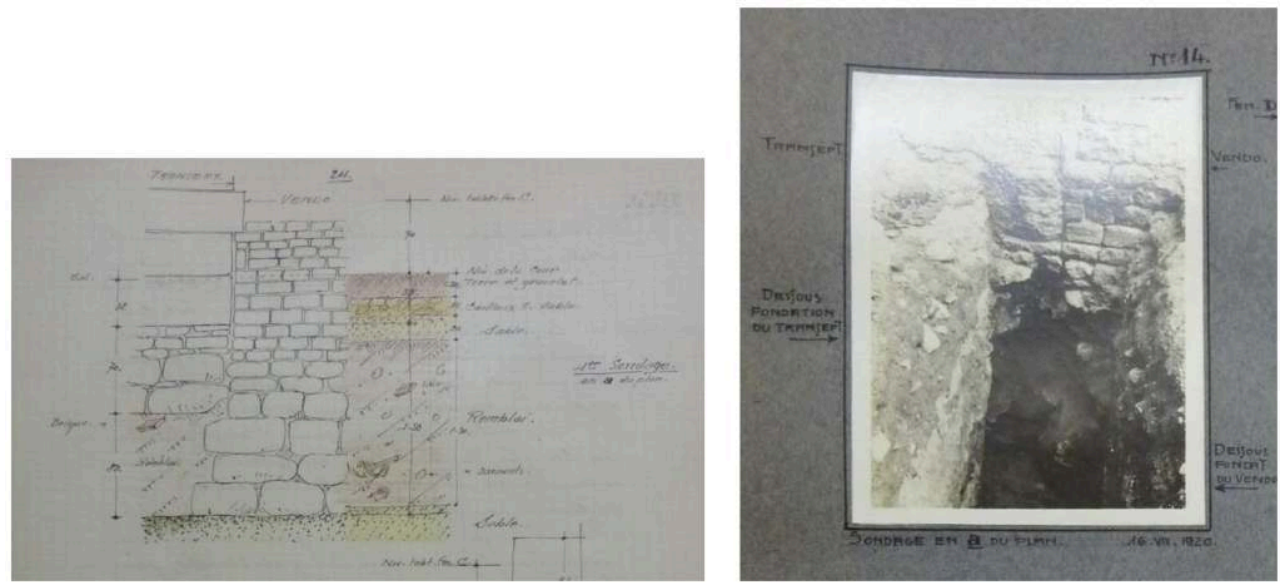

Fig. 12 - Payerne, Abbatiale. Westfassade des Kapitelsaals, Plan der Biforie nördlich des Rundbogentores. Unmasstäbliche Verkleinerung. Bild Louis Bosset.

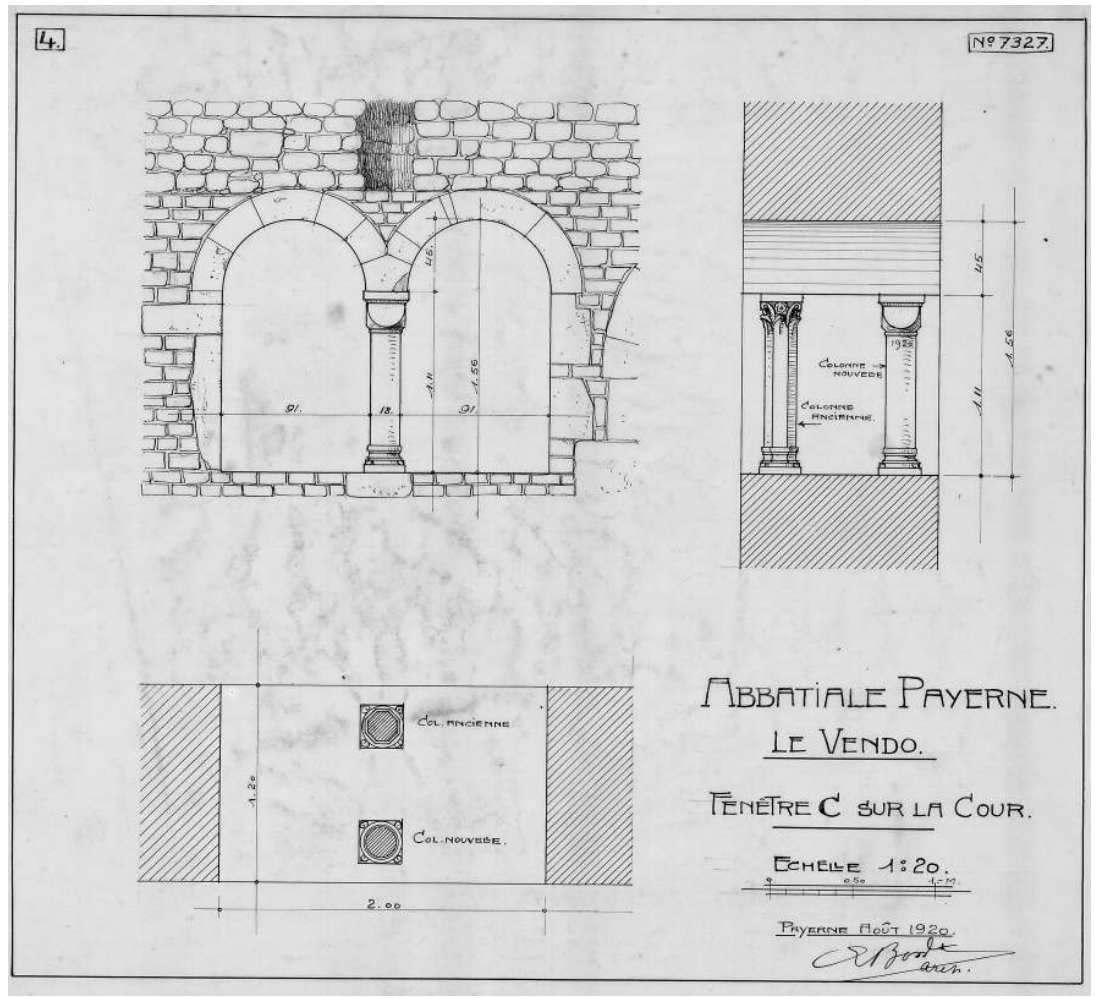

1. Auf der Westseite war in einer $1.60 \mathrm{~m}$ tiefen Sondierung eine Fuge zwischen Kapitelsaal und Querschiff zu sehen (Abb. 11). ${ }^{36}$

Erstaunlicherweise reicht entgegen aller Vermutungen die Fundamentsohle des Kapitelsaals $80 \mathrm{~cm}$ tiefer als jene des Querschiffarmes und ruht auf dem Anstehenden, während der zum monumentalen Chor gehörende Querschiffarm auf Auffüll-Material steht. Ohne dass seither die Sondierung wieder geöffnet wurde, kann vorläufig 
gefolgert werden, dass der Kapitelsaal einer älteren Bauphase angehört als Querschiff und heutiges Chor. Diese Schlussfolgerung scheint nach einer Fotografie von Bosset auf der gegenüberliegenden Seite bestätigt zu werden. Der westliche Stützpfeiler der südlichen Seitenkapelle (chapelle de Grailly) ist der Ostfassade des Vendos vorgeblendet. Stützpfeiler bzw. Seitenkapelle sind Teil des Chores, das zusammen mit dem Querschiff entstand. Sie dürften somit jünger sein als der Kapitelsaal und wohl der gesamte Ostflügel der Konventbauten, die demzufolge mit einer älteren Choranlage bestanden haben müssen.

2. Die romanische Westfassade des Kapitelsaales ist trotz vielfacher Umnutzung weitgehend erhalten geblieben. Bis auf zwei kleine queroblonge Schlitze waren alle mittelalterlichen Öffnungen zugemauert. Auf der Mittelachse wurde nun ein Rundbogenportal ${ }^{37}$ freigelegt, das von einem zwei- bzw. dreiachsigen Fenster flankiert wird. Von den Säulchen zwischen den Rundbogenfenstern fehlte jeweils die äussere. ${ }^{38}$ Die nördliche Öffnung des dreiachsigen Fensters war bereits in gotischer Zeit vermauert worden; zuvor hatte man die Säulchen entfernt und durch Sandsteine ersetzt. Das Kapitell der nördlichen Biforie darf stilistisch nach einem Vergleich mit Kapitellen aus Autun durchaus noch in die erste Hälfte des 11. Jh. datiert werden, was einen ersten Hinweis auf die Entstehungszeit des Osttraktes liefert (Abb. 13). ${ }^{39}$

Fig. 13 - Rechts: Payerne, Kapitelsaal Westseite, Biforie nördlich des Rundbogenportals. Mitte: Autun / St-Pierre l'Estrier. Rechts: Autun / Musée Rolin. Bild links: Autor. Bild Mitte: Sapin 2001 (wie Anm. 39), fig. 116. Bild rechts: Sapin 2001 (wie Anm. 39), fig. 118.
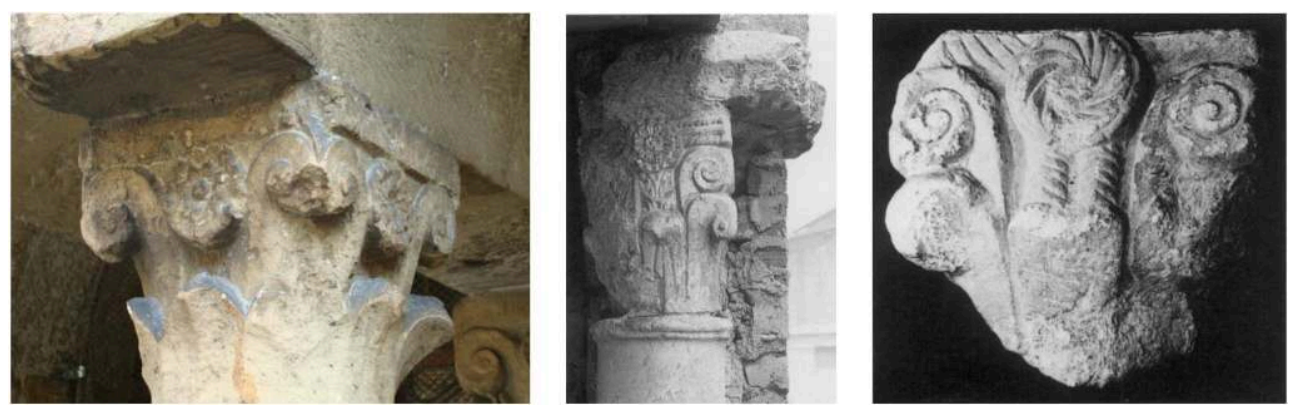

3. Die Ostseite des Kapitelsaales ist mehrphasig. Eine Fotografie, welche die Drainage entlang der Ost-Mauer zeigt, lässt unten dunkleres (feuchtes) Mauerwerk mit regelmässigem Charakter erkennen. Darüber, ab Schwellenhöhe, sind die Steine nach wie vor lagenhaft verbaut, doch weniger konsequent, was vorläufig als Möglichkeit zur Unterscheidung von zwei Bauphasen angeführt werden kann. Zwei Störungen reichen in das dunklere Mauerwerk, wovon eine im Zusammenhang mit der rechteckigen Tür des Vendo steht. Wahrscheinlich bestand hier in romanischer Zeit nie eine Tür. Bosset hat in seinen Notizen auch nie eine solche erwähnt. Wie man die ursprüngliche Befensterung zu rekonstruieren hat, ist offen. Romanische Fensterreste sind weder auf den Fotos auszumachen noch schreibt Bosset von solchen.

\section{Schlussfolgerungen}

Der durch Quellen gestützte Befund von mehreren Sakralbauten in Payerne mit Maria als Hauptpatronin mag immer noch erstaunen, auch wenn er längst bekannt ist. Doch gerade die Nennungen aus der Zeit vor 1000 lassen sich nicht mit Sicherheit einer der heutigen Kirchen von Payerne mit mittelalterlichen Wurzeln - Abbatiale und 
Paroissiale - zuweisen. Die noch nicht mit genügender Klarheit erfasste archäologische Vergangenheit der Paroissiale ist dabei ein nicht $\mathrm{zu}$ unterschätzender Faktor. Doch auch die Bauabfolge der Architekturen unter und neben der Abbatiale und selbst deren Baugeschichte müssen anhand der vorhandenen Dokumente der Grabungen von Bosset bzw. von Margot nochmals durchstudiert werden. Dies zeigt die bereits jetzt deutlich erfassbare grosse Tragweite der neuen Aufschlüsse, die aus den Notizen und Skizzen im Grabungstagebuch von Louis Bosset gewonnen werden konnten.

Fig. 14 - Payerne, Abbatiale. Schematischer Rekonstruktionsversuch des Zustandes nach dem Bau des Kapitelsaals. Schwarz: älterer Bestand. Rot: Neubau, erhalten. Hellrot: Neubau, rekonstruiert. Bild Archéotech und Autor.

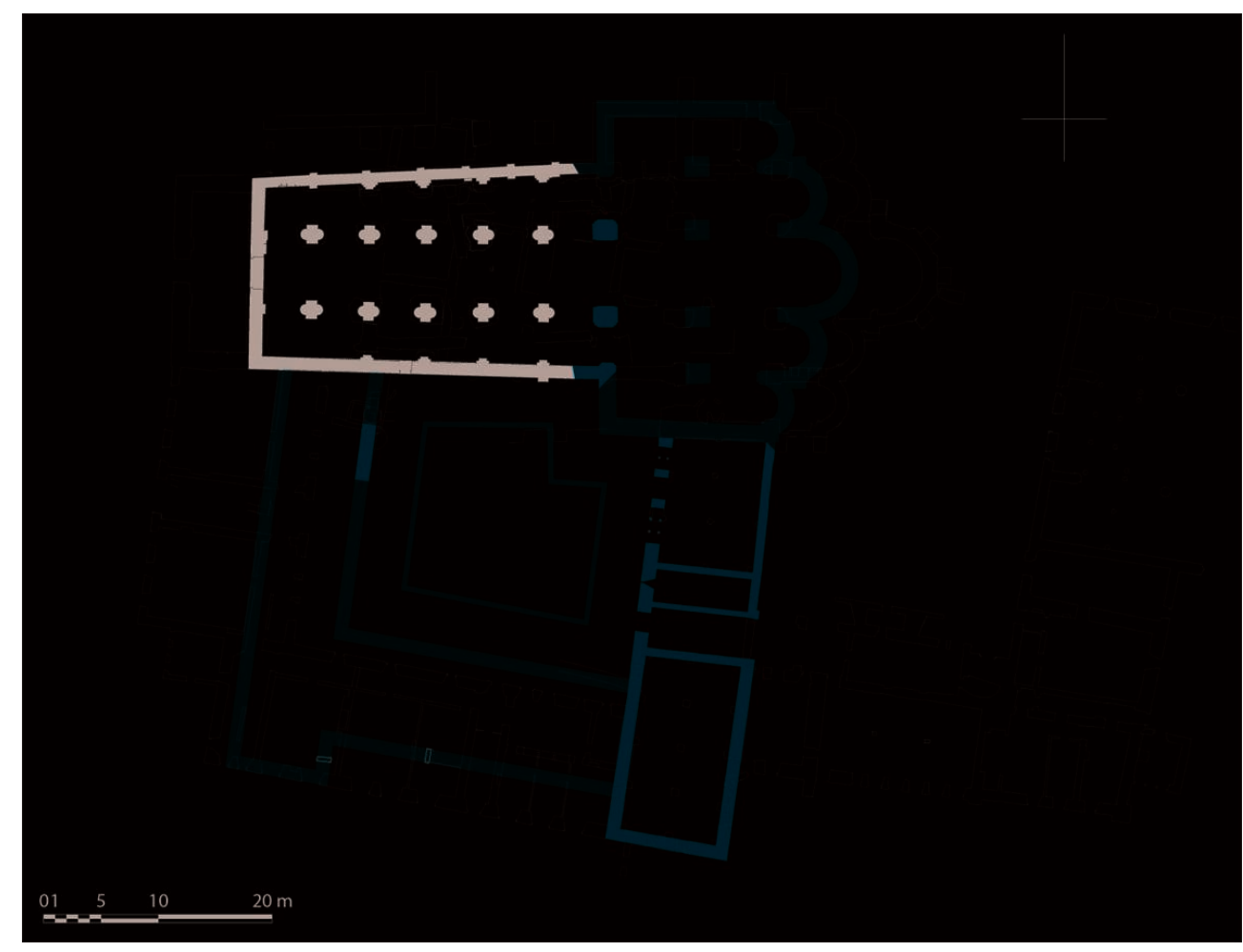

Dass das heutige Querschiff und mit ihm der Staffelchor jünger als der Kapitelsaal sind, ist anzunehmen. Der Kapitelsaal muss also zusammen mit einem anderen Chorhaupt bestanden haben (Abb.14). Dieses dürfte bis auf den Ansatz der SüdquerschiffWestmauer verloren sein, sofern es je vollendet wurde. Der ergänzte Grundriss der Klosteranlage zeigt einen Typ, der im nahen Münchenwiler ${ }^{40}$ wohl noch vor 1080/81 vollendet worden ist. Beide zeichnen sich durch einen Kreuzgang aus, dessen nordöstliche Ecke abgewinkelt ist und in den Hof einspringt, bedingt durch die gegenüber dem Querschiff nach Osten zurückversetzte Fassade des Kapitelsaals. Ob der Kapitelsaal von Payerne selbst mit dem Platz im Osten kommuniziert hatte oder nicht, ist offen. Der heutige Niveauunterschied von ca. 1,5 m zwischen Kapitelsaal und Place du Tribunal scheint eher einer Verbindung zu widersprechen.

Zwischen Abbatiale und Paroissiale liegt Friedhofsgebiet und unter der Paroissiale kamen ältere, vielleicht romanische oder noch früher errichtete Bauten zum Vorschein. Trotz dieser Befunde bleibt aber die Frage vorerst unbeantwortet, ob im Osten der Abbatiale eine zum Kloster gehörende und in die liturgischen Abläufe einbezogene Kapelle bestand. Diesen Fragen kann man sich nur nähern durch 
systematische Bearbeitung der Altgrabungen, der Schrift- und Bildquellen sowie durch erneute, wenn mögliche flächig angelegte archäologische Untersuchungen.

\section{NOTES}

1. Zur katholischen Kirche und zur reformierten Paroissiale vgl. vorerst: Jura, Jura Bernois, Neuchâtel, Vaud Genève. Guide artistique de la Suisse, 4a, version entièrement revue, corrigée et complétée, éd. par la Société d'histoire de l'art en Suisse, Berne 2011, 396-398.

2. Zur Pfarrkirche: Guide artistique Suisse, 4a (wie Anm. 1), 396-397.

3. H. E. MAYER, « Die Peterlinger Urkundenfälschungen und die Anfänge von Kloster und Stadt Peterlingen, in Deutsches Archiv für Erforschung des Mittelalters 19, Köln 1963, 30-129, 88.

4. Zum Patrozinium s. weiter unten.

Abbatiale bzw. Abteikirche sind irreführende termini, denn in den Rang einer Abtei ist der seit dem mittleren 11. Jh. als Priorat geführte Cluniazenserkonvent offiziell und allgemeingültig nie erhoben worden; vgl. Germain Hausmann, Payerne, in: Hans-Jörg Gilomen (Red.), Die Cluniazenser in der Schweiz, Helvetia Sacra (begr. von P. Rudolf Henggeler OSB, weitergeführt von Albert Bruckner), Abteilung III, die Orden mit Benediktinerregel 2, Basel / Frankfurt am Main 1991, 391-460, S. 391, 405. Eigentlich müsste die Kirche Priorale bzw. Prioratskirche genannt werden. Bis zur Mitte des 11. Jh. unterstand der Konvent von Payerne direkt den Äbten von Cluny.

5. Zu den Konventbauten vgl. vorerst Guide artistique Suisse 4a (wie Anm. 1), 396.

6. E. SECRETAN, «François-Rodolphe de Dompierre: un lieutenant-colonel archéologue 1775-1844 ", in Revue Historique Vaudoise 16 (1908), p. 289-302, p. 321-337, hier besonders p. 297. De Dompierre, ein gebürtiger Payernois, war ab 1822 Conservateur des antiquités für den Norden des Kantons Waadt.

7. Louis Bosset, ein gebürtiger Payernois, übernahm 1934 von Architekt Albert Naef das Amt des Kantonsarchäologen, das er bis zu seinem Tod inne hatte.

8. Dokumentationen in den Archives Cantonales Vaudoises (ACV) und in den Archives Communales de Payerne (ACPY).

9. Diese grösstenteils von der Association pour la restauration de l'Abbatiale finanzierte Dokumentation lagert in Zurzach im Privat-Archiv von Hans Rudolf Sennhauser und wurde für die aktuellen Arbeiten nicht zur Verfügung gestellt.

10. P. EGgEnberger, X. MUNGER, «Payerne, District de Payerne, Résumé des résultats des investigations archéologiques en 1989/1990, Chronique des fouilles archéologiques, 1990 », in Revue historique vaudoise (1991), p. 173-176; P. EGGENBERGER, X. MUNGER, « Payerne, District de Payerne, Les investigations archéologiques complémentaires à l'église paroissiale de Payerne en 1991, Chronique des fouilles archéologiques, 1991 ", in Revue historique vaudoise (1992), p. 222-227 ; P. EGGENBERGER, X. MUNGER, Payerne, District de Payerne, église paroissiale, Investigations dans la nef, Chronique des fouilles archéologiques, 1992, in Revue historique vaudoise (1993), p. 192-198; Grabungsdokumentationen in ACV (wie Anm. 8).

11. J.-D. Blavignac, Histoire de l'architecture sacrée du quatrième au dixième siècle dans les anciens évêchés de Genève, Lausanne et Sion. Text- und Tafelband. Paris, Londres, Leipzig 1853.

12. J. R. RAHN, L'église abbatiale de Payerne, traduit de l'allemand par William Cart, Lausanne 1893.

13. L. BOSSET, L'église abbatiale de Payerne, Payerne 1917, 2. 
14. L'Abbatiale de Payerne, Société Académique Vaudoise (éd), préf. de Colin Martin, Bibliothèque Historique Vaudoise XXXIX, Lausanne 1966.

15. G. Zarnecki, «La sculpture de Payerne», in Abbatiale de Payerne, 1966 (wie Anm. 14), p. 139-164.

16. H. R. SENNHAUSER, Romainmôtier und Payerne. Studien zur Cluniazenserarchitektur des 11. Jahrhunderts in der Westschweiz, Basel 1970.

17. H. R. SENNHAUSER, Die Abteikirche von Payerne, Schweizerische Kunstführer, hg. von der Gesellschaft für Schweizerische Kunstgeschichte, Serie 50, Nr. 495, Bern 1991 ; Die erste Ausgabe des Kunstführers stammt vom Kunst- und Architekturhistoriker Hans Reinhardt (1902-1984) :

H. REINHARDT, L'Abbatiale de Payerne, Guides des Monuments suisses, publiés par la Société d'Histoire de l'Art en Suisse, Berne 1953.

18. Zur Geschichte vgl. HAUSMANN 1991 (wie Anm. 4).

19. HAUSMANN 1991 (wie Anm. 4), 431.

20. JOTSALDUS, de vita et virtutibus sanct Odilonis abbatis, in Patrologia Latina, in J. P. MIGNE (éd.), 142, Sp. 897-940 ; Cartulaire du chapitre de Notre-Dame de Lausanne, in Ch. ROTH (éd.), MDR, $3^{\text {e }}$ sér., 3 , Lausanne 1948.

21. Das Pseudo-Testament wurde auf den 1. April 961 datiert. Es liegen zwei Versionen vor: die eine aus dem frühen 12. Jh. (ACV [wie Anm. 8], C I a 3), die andere aus dem mittleren 12. Jh. (AEFribourg (Payerne 1, 1.4. 961). MGH DD Reg. Burg. 190-201, Nr. 55. Vgl. Hausmann 1991 (wie Anm. 4), 392 und Anm. 14. - Anfang des 20. Jh. wurde erkannt, dass es sich bei den beiden Dokumenten nicht um die Originale handelt (Mayer 1963 [wie Anm. 3], 50 und Anm. 76).

22. Der 24. Juni ist Tag der Geburt Johannis des Täufers.

23. Cartulaire de Lausanne (wie Anm. 20), 23, Nr. 16d. Vgl. Auch Mayer 1963 (wie Anm. 3), 34.

24. MAYER, 1963 (wie Anm. 3), 36.

25. MGH DD Reg. Burg. Nr. 35.

26. MAYER 1963 (wie Anm. 3), 74.

27. MAYER 1963 (wie Anm. 3), 38, 74.

28. MGH SS 4, 641 ; MGH DD Reg. Burg., 149, Nr. 35.

29. Erst im frühen 16. Jh. wird erwähnt, dass das Grab von Berta im Chorbereich der Abbatiale liegt. MAYER, 1963 (wie Anm. 3), 45.

30. MGH DD Reg. Burg, Nr. 35, 149.

31. MGH SS 4, 641.

32. MGH DD Otto II., 60-61, Nr. 51. Hausmann 1991 (wie Anm. 4), 391.

33. Patrozinium: Guide artistique Suisse 4a (wie Anm. 1), 396. 1183 : MAYER 1963 (wie Anm. 3), 88.

34. Zur Entstehung der Stadt Payerne vgl. MAYER 1963 (wie Anm. 3), 78-90.

35. Das bebilderte Grabungstagebuch (L. BOSSET, «Église abbatiale de Payerne. Restauration » in Journal des Travaux. MS, Payerne 1920) befindet sich ebenso im Gemeindearchiv von Payerne wie die Grabungsfotos und die masstäblichen Pläne.

36. BOSSET, 1920 (wie Anm. 35), S. 4, 19, 24, 25, 30 / Foto 14.

37. BOSSET, 1920 (wie Anm. 35), $4:$ «... la porte romane, démurée le 20 avril pour la sortie des tonneaux... ".

38. BOSSET, 1920 (wie Anm. 35), 4.

39. C. SAPIN, Les prémices de l'art roman en Bourgogne, Auxerre, 2001.

40. P. EGGENBERGER, G. KECK et al., Schloss Münchenwiler - ehemaliges Cluniazenser-Priorat, Bericht über die Grabungen und Bauuntersuchungen von 1986-1990, Bern 2000. 


\section{RÉSUMÉS}

Die seit dem frühen 19. Jahrhundert mit Unterbrüchen andauernde archäologische Erforschung der Abbatiale von Payerne und kunsthistorische Studien seit der zweiten Hälfte des 19. Jahrhunderts unterstreichen die grosse Bedeutung der Abbatiale innerhalb der frühen romanischen Architektur. Seit langem ist bekannt, dass im späten 6. Jh. ein Marienoratorium in Payerne geweiht wurde. Es ist aber bislang nicht lokalisiert. U.a. wegen des stark reduzierten Quellenbestandes bleibt der Hergang der Gründung des cluniazensischen Klosters in der zweiten Hälfte des 10. Jahrhunderts weitgehend im Dunkeln. Ebenso wenig ist eruierbar, ob eine Marienkapelle östlich des Kapitelsaales bestand. Jüngste Studien zeigen auf, dass der Kapitelsaal älter als das heutige Querhaus bzw. die bestehende Choranlage ist. Sein Architekturschmuck datiert wohl in die erste Hälfte des 11. Jahrhunderts. Zusammen mit den übrigen bekannten Elementen lässt sich ein Klostergrundriss rekonstruieren, dem jener von Münchenwiler in Grundzügen gleicht.

La recherche archéologique et les études sur l'histoire architecturale de l'abbatiale de Payerne menées dès le XIX ${ }^{e}$ siècle, montrent toute l'importance de cette église dans l'architecture sacrée romane précoce. Depuis longtemps, on sait qu'à Payerne existait au $\mathrm{VI}^{\mathrm{e}}$ siècle tardif un oratoire dédié à Sainte-Marie. Celui-ci n'a pas été localisé jusqu'à présent. Entre autres, à cause du fond très réduit des sources écrites, l'acte de la fondation du monastère clunisien dans la deuxième moitié $\mathrm{du} \mathrm{x}^{\mathrm{e}}$ siècle reste à quelques détails près encore obscur. De même, il n'a pas été élucidé s'il existait autrefois une chapelle mariale à l'est de la salle capitulaire. Des études récentes démontrent, que la salle capitulaire est plus ancienne que le transept et le chevet existant. Le décor architectural de la salle date vraisemblablement de la première moitié du $\mathrm{xI}^{\mathrm{e}}$ siècle. Avec les autres éléments architecturaux, le plan d'ensemble du monastère peut être reconstitué ; il présente des ressemblances avec celui de Villars-les-Moines.

\section{INDEX}

Index géographique : Suisse/Payerne

Mots-clés : abbatiale, Marius d'Avenches, fondation du couvent, salle capitulaire, cloître, vocable Sainte-Marie 